\title{
Urethral Stricture
}

National Cancer Institute

\section{Source}

National Cancer Institute. Urethral Stricture. NCI Thesaurus. Code C79821.

Narrowing of the lumen of the urethra. 